\title{
The Student Dust Counter: Status report at 23 AU
}

\author{
Jamey R. Szalay, Marcus Piquette, and Mihály Horányi \\ Laboratory for Atmospheric and Space Physics, and Department of Physics, University of Colorado, Boulder, CO 80303, U.S.A.
}

(Received November 7, 2012; Revised February 19, 2013; Accepted February 19, 2013; Online published October 24, 2013)

\begin{abstract}
The Student Dust Counter (SDC) is an impact dust detector on board the New Horizons Mission to Pluto. SDC was designed to resolve the mass of dust grains in the range of $10^{-12}<m<10^{-9} \mathrm{~g}$, covering an approximate size range of $0.5-10 \mu \mathrm{m}$ in particle radius. The measurements can be directly compared to the prediction of a grain trajectory tracing model of dust originating from Edgeworth-Kuiper Belt (EKB). Comparing our measurements and model prediction is used to estimate the net mass production rate and the ejecta mass distribution power law exponent to be $\dot{M}=(8.9 \pm 0.5) \times 10^{5} \mathrm{~g} / \mathrm{s}$ and $\alpha=3.02 \pm 0.04$, respectively. Through April 2012 , the New Horizons spacecraft reached approximately $23 \mathrm{AU}$, enabling SDC to map the dust density distribution along its path.
\end{abstract}

Key words: Interplanetary dust, instrumentation, Pluto, Edgeworth-Kuiper belt.

\section{Introduction}

The Student Dust Counter (SDC) is an impact dust detector on board the New Horizons Mission to Pluto. It is designed to map the spatial and size distribution of interplanetary dust particles in order to verify the existence of the predicted structures in our interplanetary dust disk. Table 1 lists the five spacecraft that have carried dust detectors beyond the asteroid belt: Pioneers 10 and 11 (Humes, 1980), Galileo (Grün et al., 1992a), Ulysses (Grün et al., 1992b), and Cassini (Srama et al., 2004). SDC provides the first dust measurements beyond $18 \mathrm{AU}$, where the Pioneer sensors stopped working. After the Pluto-Charon fly-by, SDC will continue to measure dust as it transits through the Edgeworth-Kuiper belt. These observations will advance our understanding of the origin and evolution of our own solar system and allow for comparative studies of planet formation in dust disks around other stars. SDC is the only experiment to date that was designed, built, and is now operated by students on a deep space mission. It has provided an unparalleled opportunity for about 25 students to learn about space instrumentation. SDC continues to involve an ever changing smaller group of 3 students who operate the instrument and analyze the data, handing over their responsibility to the next 'generation' every few years. SDC could not have been built without the support of the late Dr. Tony Tuzzolino who first designed these types of instruments (Tuzzolino, 1991, 1992, 1996).

In Section 2 we briefly review the scientific motivation for SDC, followed by the instrument description in Section 3, and report on the observations to date in Section 4. The estimates on the dust production in the EdgeworthKuiper belt based on SDC and Pioneer 10 measurements

Copyright (C) The Society of Geomagnetism and Earth, Planetary and Space Sciences (SGEPSS); The Seismological Society of Japan; The Volcanological Society of Japan; The Geodetic Society of Japan; The Japanese Society for Planetary Sciences; TERRAPUB.

doi:10.5047/eps.2013.02.005 are discussed in Section 5.

\section{Science Background}

The interplanetary dust particle (IDP) population reflects a complex balance between sources, transport, and sinks which are all functions of the dust particle sizes and their positions in the solar system. IDPs are created primarily in asteroid-asteroid collisions, from the disintegration of comets near the Sun, from collisions between Kuiper Belt objects (KBOs), and from the continuous meteoroid bombardment of the moons, rings, asteroids and KBOs. Data from near-Earth and deep space missions suggest five distinct IDP populations (Divine, 1993). While this model makes predictions for IDP populations throughout the solar system, it has not been validated by direct measurements beyond 18 AU. The Divine (1993) model was used to set the science and measurement requirements for SDC in 2004. Since then, newer and more complete models became available (Kuchner and Stark, 2010; Vitense et al., 2010, 2012; Han et al., 2011b).

Beginning at about $3 \mathrm{AU}$ from the Sun, the Pioneer 10 and 11 observations of particles larger than about $10 \mu \mathrm{m}$ in radius showed a constant spatial density (number of particles per unit volume) out to 18 AU (Humes, 1980). At this distance, the argon and nitrogen gas mixture in the Pioneer pressurized detector cells froze and no further measurements could be made.

IDPs which are born on the outskirts of the solar system slowly lose orbital energy due to Poynting-Robertson drag and migrate toward the Sun. A $10 \mu \mathrm{m}$ radius grain born at 50 AU reaches the inner solar system in about 6.5 My. However, the continuous migration can significantly slow down or come to a halt due to mean motion resonances with the planets (Liou and Zook, 1999; Moro-Martín and Malhotra, 2002, 2003). Grains can also get ejected from the solar system via close encounters with the planets (Liou and Zook, 1999). Orbital integrations find that Neptune 
Table 1. In situ dust measurements beyond the asteroid belt.

\begin{tabular}{|c|c|c|}
\hline Spacecraft & Max Heliocentric Distance [AU] & Detector area $\left[\mathrm{m}^{2}\right]$ \\
\hline Pioneer 10 & 18 & $<0.26$ \\
\hline Pioneer 11 & 10 & $<0.57$ \\
\hline Ulyssses & 5.4 & 0.1 \\
\hline Galileo & 5.2 & 0.1 \\
\hline Cassini & 9.5 & 0.1 \\
\hline SDC & 50 & 0.1 \\
\hline
\end{tabular}
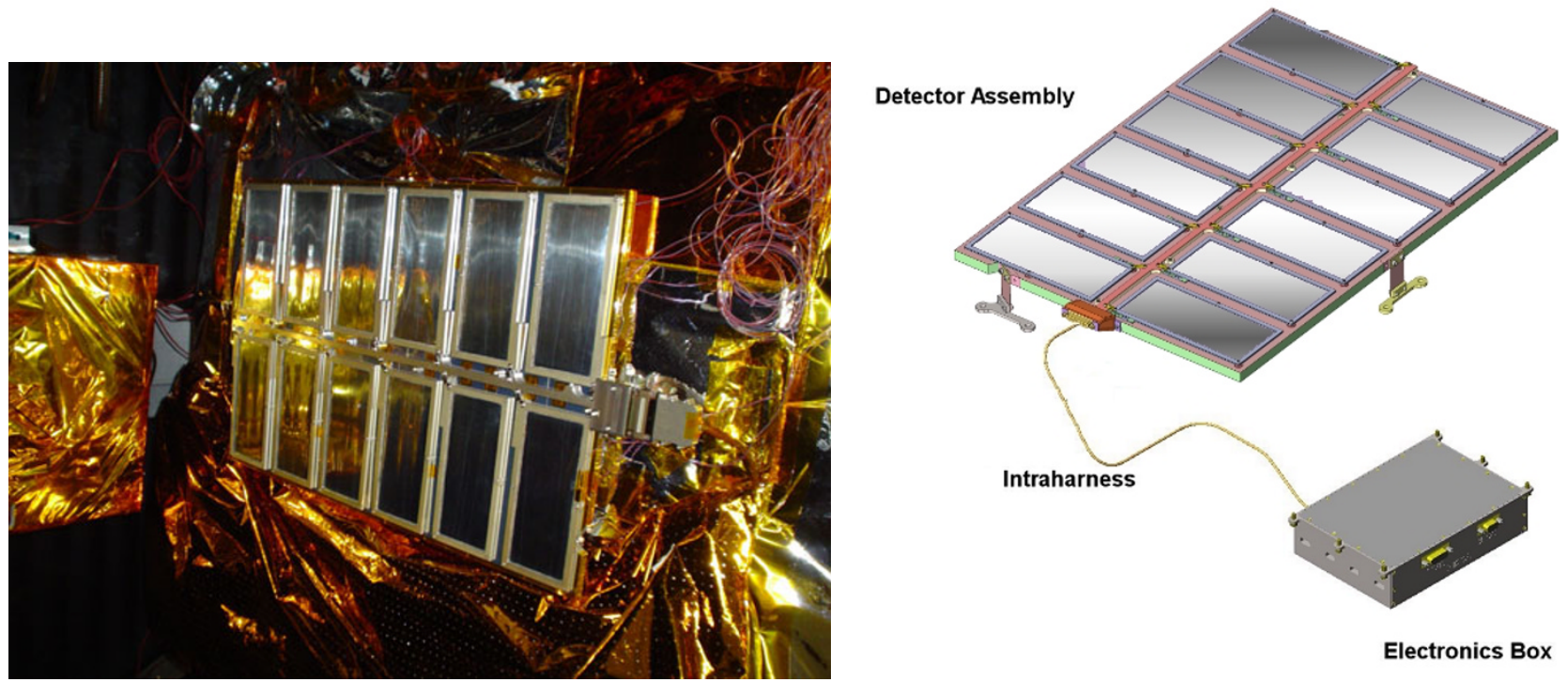

Fig. 1. SDC during thermal testing (left), and the schematic drawing of the experiment (right). Two additional sensor patches (not shown) are attached to the backside of the detector assembly to monitor noise events. SDC $\sim 0.1 \mathrm{~m}^{2}$ of sensitive area. It was designed to measure the mass of IDPs in the range of $10^{-12}$ to $10^{-9} \mathrm{~g}$. Impacts of bigger dust particles are registered the without the ability to determine their mass. The instrument weighs 1.6 $\mathrm{kg}$ and consumes 5.1 watts of average power.

prolongs the lifetime of the inwardly migrating dust grains outside its orbit, imprinting its resonance structure on the spatial density of dust in the Edgeworth-Kuiper belt.

Jupiter, on the other hand, acts as a gate-keeper and keeps the inner part of our solar system relatively dust free by ejecting most of the particles that would cross its orbit. The nature of the inner planets effect on dust distribution is largely hidden due to dust production from active comets.

SDC measurements made as New Horizons crosses the solar system will help to understand the generation, the transport, and the loss processes of IDPs. The measurements of the density variations outside the orbit of Jupiter will allow for estimating the rate of dust production in the Kuiper Belt, the collisional history of the region, and the mass distribution of the primordial KBO population (Stern, 1996).

\section{Instrument Description}

Figure 1 shows the SDC instrument; here we only briefly summarize its working principles, the full technical details of the instrument are described in Horányi et al. (2008). The SDC instrument consists of a set of polyvinylidene fluoride (PVDF) film impact sensors, carried on a detector support panel, which is mounted on the exterior of the New Horizons spacecraft. It is outside the spacecraft multi-layer insulating blanket, facing the ram direction. Signals from the sensors are collected through an intra-harness that runs from the detector assembly into the spacecraft interior to the instrument electronics box mounted opposite the detector panel.

The measurement requirements of the SDC instrument were established by estimating the particle mass detection limit needed to resolve the expected features of the dust distribution. The characteristic width of the predicted resonance structures is on the order of $1 \mathrm{AU}$, hence a spatial resolution of $\approx 0.1 \mathrm{AU}$ assures adequate sampling. With the characteristic spacecraft speed of $\approx 13 \mathrm{~km} / \mathrm{s}$ during cruise, this is equivalent to an integration period of one week. Based on the current models (Divine, 1993), a detector with a lower mass detection limit of about $10^{-12} \mathrm{~g}$ and $0.1 \mathrm{~m}^{2}$ surface area is predicted to have on the order of 1 IDP hit per week outside of dust structures. SDC was designed to resolve the mass of IDPs in the range of $10^{-12}<m<10^{-9} \mathrm{~g}$ within factors of $<2$, covering an approximate size range of $0.5-5 \mu \mathrm{m}$ in particle radius. Bigger grains are also recorded without the ability of resolving their mass.

To process SDC data, all grains are assumed to be on circular, Keplerian orbits. For each detected impact, an impact angle is calculated between the grain and SDC area vector. Impacts with angles greater than $45^{\circ}$ are rejected as noise since the charge generated by such an impact would be below our detection limit. From the set of valid impacts, 


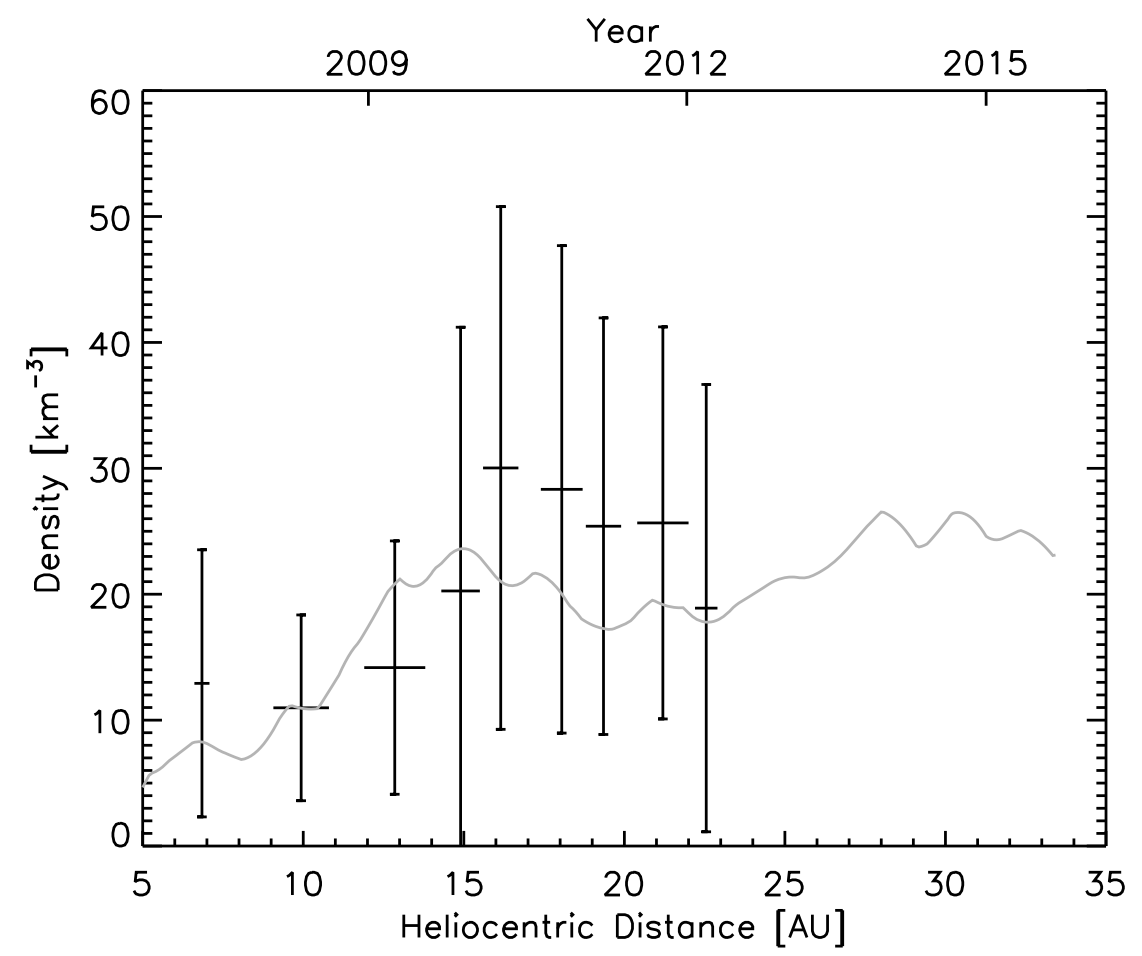

Fig. 2. SDC measurements of the dust flux $\left(m>10^{-12} \mathrm{~g}\right)$ through April 2012. The continuous line represents a model prediction out to the Pluto encounter at $33 \mathrm{AU}$ based on SDC and Pioneer 10 data taken between approximately 5 and $18 \mathrm{AU}$ (Han et al., 2011a).

measured dust grain fluxes are calculated. Densities are calculated from these fluxes using the circular orbit assumption and local spacecraft velocity for each impact.

\section{SDC Measurements Through 2012}

When SDC data are received on Earth, they are sent to the New Horizons mission operations center at the Johns Hopkins University Applied Physics Laboratory. The SDC data, as well as several ancillary spacecraft data packets, are also sent to SDCs home institution, the Laboratory for Atmospheric and Space Physics (LASP). Simultaneously, it is also received at the Tombaugh Science Operations Center (TSOC) of the Southwest Research Institute (SwRI) in Boulder. The data are sent through "levels" of processing software that progressively decode more of the packets and send the decoded data to a database. The SDC final science product includes noise corrected impact rates as function of mass (Horányi et al., 2008).

The SDC measurements made inside 5 AU were compared to previous interplanetary and interstellar dust measurements by the Ulysses and Galileo DDS instruments. Taking into account the instrument sensitivities, viewing areas and pointing, the IDP fluxes in the inner solar system across the three instruments were found to be in good agreement (Kobayashi et al., 2010; Poppe et al., 2010b). The SDC measured densities, converted from fluxes measured outside the orbit of Jupiter through to the time of this analysis at $23 \mathrm{AU}$, are shown in Fig. 2.

\section{Comparison to Model Results}

SDC data provide a unique opportunity to validate model results of the IDP distribution past 18 AU with direct measurements. The model we use to compare SDC data to is de- scribed in Han et al. (2011a). Briefly, we traced dust grains over a range of grain radii from the EKB throughout the solar system under the influence of gravity, solar radiation pressure and solar wind drag in order to establish a statistical equilibrium density distribution for each grain size. All grains in this model were started in the region $35<r_{s}<50$ $\mathrm{AU}$, with the orbital elements randomly selected from those of three categories of KBOs in the ratio of Classical : Scattered : Plutinos $=0.67: 1.00: 0.25$ (Kavelaars et al., 2009). Integration of the grain trajectory was stopped when a grain arrived near the Sun $\left(r_{s}<0.1 \mathrm{AU}\right)$ or left the solar system $\left(r_{s}>200 \mathrm{AU}\right)$ with net positive orbital energy.

By keeping track of each grain's position, a relative, statistical equilibrium density map can be calculated for each grain size. The density distribution is constructed by calculating the total time all particles spent in each part of the solar system. A large fraction of the grains are found to be in mean-motion resonance (MMR) with Neptune, where the ratios of the orbital periods of Neptune and the grains are the ratio of two integers. In order to highlight the role that MMR with Neptune play in shaping the equilibrium density, all trajectories are transformed to the Neptune corotating frame. As an example, Fig. 3 shows the combined column density for grains with radius $10 \mu \mathrm{m}$. The white line shows the trajectory of New Horizons integrated out to the Pluto encounter at $33 \mathrm{AU}$. The spatial structures are more pronounced for larger particles, as their radial migration due to Poynting-Robertson drag is slower than that for smaller grains, hence their residence time in MMRs are longer, leading to significant density enhancements.

To date, the two dedicated measurements of interplanetary dust grain density in the outer solar system come from SDC (Horányi et al., 2008; Stern, 2008; Poppe et al., 


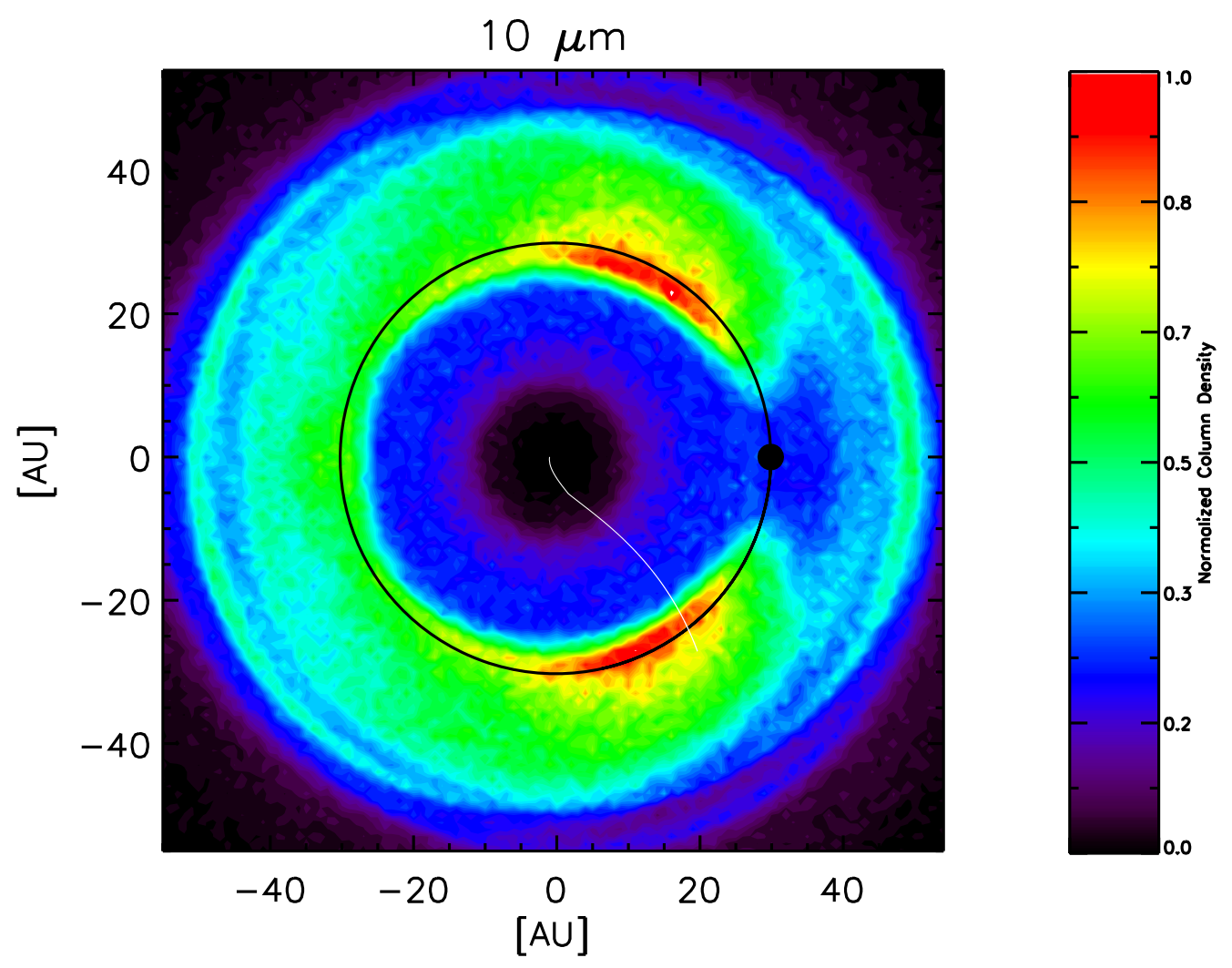

Fig. 3. Modeled column density of $10 \mu \mathrm{m}$ dust within $\pm 0.5 \mathrm{AU}$ of the ecliptic plane, rotated into the Neptune co-rotating frame to illuminate the structure of the disk (Han et al., 2011a). The white line shows the New Horizons trajectory integrated out to the Pluto encounter at 33 AU. Neptune and its orbit are shown by the black circle and line respectively. Note: The large majority of grain impacts to SDC will be $\sim 0.5 \mu \mathrm{m}$. This plot was chosen to illustrate the significant MMR structure, which is more pronounced for larger grains.

2010a) and the meteoroid detector on the Pioneer 10 spacecraft (Humes, 1980). Both SDC and Pioneer 10 flux measurements are converted to densities by dividing out the theoretical dust grain impact velocity as a function of heliocentric distance, as described in the Instrument Description.

In order to compare the model-predicted densities with the experimental measurements, the model densities at each grain radius were added together after appropriately weighting by the assumed power law distribution (Stern, 1996; Yamamoto and Mukai, 1998), $d \dot{M} / d m=\dot{M}_{o}\left(m / m_{o}\right)^{-\alpha / 3}$, where $d \dot{M} / d m$ is the differential mass production rate of dust grains between $m$ and $d m$ in the Edgeworth-Kuiper belt, $\dot{M}_{o}$ is a normalization constant, and $m_{o}=10^{-11} \mathrm{~g}$. The net mass production rate in the Edgeworth-Kuiper belt, $\dot{M}$ $[\mathrm{g} / \mathrm{s}]$, is found by integrating the distribution from $m_{\min }=$ $10^{-14} \mathrm{~g}\left(a_{d}=0.1 \mu \mathrm{m}\right)$ to $m_{\max }=10^{-8} \mathrm{~g}\left(a_{d}=10\right.$ $\mu \mathrm{m})$ (Stern, 1996; Yamamoto and Mukai, 1998). The data was compared to the model by using a two-parameter $\chi^{2}$ goodness-of-fit function. Varying both $\dot{M}$ and $\alpha$, the minimum $\chi^{2}$ value was found to occur at $\dot{M}=(8.9 \pm 0.5) \times 10^{5}$ $\mathrm{g} / \mathrm{s}$ and $\alpha=3.02 \pm 0.04$ (Han et al., 2011a), with the mass production rate well within previous theoretical estimates (Stern, 1996; Yamamoto and Mukai, 1998). Using the bestfit values for the mass production rate and the power law index, the model predicted densities can be compared with the observations, as shown by the blue line in Fig. 2. Naturally, these estimates could be greatly improved if there was another set of measurements along a different path than New Horizons, cutting through our solar system in a differ- ent direction. For completeness we note, that beyond Saturn a significant part of the dust population is expected to be from interstellar dust particles. The characteristic radius of these grains is on the order of $0.3 \mu \mathrm{m}$, and they remain undetected by SDC (Landgraf, 2000; Sterken et al., 2012).

In addition to the previously mentioned dust grain populations, SDC will also be flying though the Pluto-Charon dust cloud as it approaches the Pluto-Charon system. Recent simulations show this impact-generated dust cloud contains grains ejected from Pluto and its moons and predicted fluxes for SDC are $\sim 10^{-4} \mathrm{~m}^{-2} \mathrm{~s}^{-1}$ (Poppe and Horányi, 2011).

\section{Conclusions}

Based on in-flight test and calibrations, and the science data as of to date, we expect SDC to continue to make dust measurements beyond Pluto. The Pluto encounter will take place in July 2015, at a distance of 32 AU from the Sun. New Horizons is expected to remain fully functional and operate for decades to come, reaching deep into the Edgeworth-Kuiper belt. Combining interplanetary dust grain measurements by SDC with a dynamical dust grain tracing code, enabled us to estimate the mass production rate $\dot{M}=(8.9 \pm 0.5) \times 10^{5} \mathrm{~g} / \mathrm{s}$ and power law index of the initial size distribution $\alpha=3.02 \pm 0.04$ (Han et al., 2011a). SDC will continue to provide insight about the dust production at the outskirts of our Solar system and enable comparisons with dust disks around other stars. 
Acknowledgments. This work was supported by the New Horizons Mission. Dr. A. Poppe, now at UC Berkeley, was the previous lead graduate student to oversee the operations of SDC.

\section{References}

Divine, N., Five populations of interplanetary meteoroids, J. Geophys. Res., 98, 17029-17048, doi:10.1029/93JE01203, 1993.

Grün, E., H. Fechtig, M. S. Hanner, J. Kissel, B.-A. Lindblad, D. Linkert, D. Maas, G. E. Morfill, and H. A. Zook, The galileo dust detector, Space Sci. Rev., 60, 317-340, doi:10.1007/BF00216860, 1992a.

Grün, E., H. Fechtig, J. Kissel, D. Linkert, D. Maas, J. A. M. McDonnell, G. E. Morfill, G. Schwehm, H. A. Zook, and R. H. Giese, The ULYSSES dust experiment, Astron. Astrophys. Suppl., 92, 411-423, $1992 \mathrm{~b}$.

Han, D., A. R. Poppe, M. Piquette, E. Grün, and M. Horányi, Constraints on dust production in the Edgeworth-Kuiper Belt from Pioneer 10 and New Horizons measurements, Geophys. Res. Lett., 38, L24102, doi:10.1029/2011GL050136, 2011a.

Han, D., A. R. Poppe, M. Piquette, E. Grün, and M. Horányi, Constraints on dust production in the Edgeworth-Kuiper Belt from Pioneer 10 and New Horizons measurements, Geophys. Res. Lett., 38, L24102, doi:10.1029/2011GL050136, 2011 b.

Horányi, M., V. Hoxie, D. James, A. Poppe, C. Bryant, B. Grogan, B. Lamprecht, J. Mack, F. Bagenal, S. Batiste, N. Bunch, T. Chanthawanich, F. Christensen, M. Colgan, T. Dunn, G. Drake, A. Fernandez, T. Finley, G. Holland, A. Jenkins, C. Krauss, E. Krauss, O. Krauss, M. Lankton, C. Mitchell, M. Neeland, T. Reese, K. Rash, G. Tate, C. Vaudrin, and J. Westfall, The student dust counter on the New Horizons mission, Space Sci. Rev., 140, 387-402, doi:10.1007/s11214-007-9250-y, 2008.

Humes, D. H., Results of Pioneer 10 and 11 meteoroid experimentsInterplanetary and near-Saturn, J. Geophys. Res., 85, 5841-5852, doi:10.1029/JA085iA11p05841, 1980.

Kavelaars, J. J., R. L. Jones, B. J. Gladman, J. M. Petit, J. W. Parker, C. Van Laerhoven, P. Nicholson, P. Rousselot, H. Scholl, O. Mousis, B. Marsden, P. Benavidez, A. Bieryla, A. Campo Bagatin, A. Doressoundiram, J. L. Margot, I. Murray, and C. Veillet, The Canada-France ccliptic plane survey-L3 data release: The orbital structure of the Kuiper belt, Astron. J., 137(6), 4917-4935, 2009.

Kobayashi, H., H. Kimura, S. Yamamoto, S. I. Watanabe, and T. Yamamoto, Ice sublimation of dust particles and their detection in the outer solar system, Earth, 62(1), 57-61, 2010.

Kuchner, M. J. and C. C. Stark, Collisional grooming models of the Kuiper belt dust dloud, Astron. J., 140, 1007-1019, doi:10.1088/00046256/140/4/1007, 2010.

Landgraf, M., Modeling the motion and distribution of interstellar dust inside the heliosphere, J. Geophys. Res., 105, 10303-10316, doi:10.1029/1999JA900243, 2000.

Liou, J.-C. and H. A. Zook, Signatures of the giant planets imprinted on the Edgeworth-Kuiper belt dust disk, Astron. J., 118(1), 580-590, 1999.

Moro-Martín, A. and R. Malhotra, A study of the dynamics of dust from the Kuiper belt: Spatial distribution and spectral energy distribution, Astron. J., 124, 2305-2321, doi:10.1086/342849, 2002.

Moro-Martín, A. and R. Malhotra, Dynamical models of Kuiper belt dust in the inner and outer solar system, Astron. J., 125, 2255-2265, doi:10.1086/368237, 2003.

Poppe, A. and M. Horányi, The effect of Nix and Hydra on the putative Pluto-Charon dust cloud, Planet. Space Sci., 59, 1647-1653, doi:10.1016/j.pss.2011.08.001, 2011.

Poppe, A., B. Jacobsmeyer, D. James, and M. Horányi, Simulation of polyvinylidene fluoride detector response to hypervelocity particle impact, Nucl. Instr. Meth. Phys. Res. A, 622, 583-587, doi:10.1016/j.nima.2010.07.088, 2010a.

Poppe, A., D. James, B. Jacobsmeyer, and M. Horányi, First results from the Venetia Burney Student Dust Counter on the New Horizons mission, Geophys. Res. Lett., 37, L11101, doi:10.1029/2010GL043300, 2010b.

Srama, R., T. J. Ahrens, N. Altobelli, S. Auer, J. G. Bradley, M. Burton, V. V. Dikarev, T. Economou, H. Fechtig, M. Görlich, M. Grande, A. Graps, E. Grün, O. Havnes, S. Helfert, M. Horanyi, E. Igenbergs, E. K. Jessberger, T. V. Johnson, S. Kempf, A. V. Krivov, H. Krüger, A. Mocker-Ahlreep, G. Moragas-Klostermeyer, P. Lamy, M. Landgraf, D. Linkert, G. Linkert, F. Lura, J. A. M. McDonnell, D. Möhlmann, G. E. Morfill, M. Müller, M. Roy, G. Schäfer, G. Schlotzhauer, G. H. Schwehm, F. Spahn, M. Stübig, J. Svestka, V. Tschernjawski, A. J. Tuzzolino, R. Wäsch, and H. A. Zook, The Cassini cosmic dust analyzer, Space Sci. Rev., 114, 465-518, doi:10.1007/s11214-004-1435-z, 2004.

Sterken, V. J., N. Altobelli, S. Kempf, G. Schwehm, R. Srama, and E. Grün, The flow of interstellar dust into the solar system, Astron. Astrophys., 538, A102, doi:10.1051/0004-6361/201117119, 2012.

Stern, S. A., Signatures of collisions in the Kuiper Disk, Astron. Astrophys., 310, 999-1010, 1996.

Stern, S. A., The new horizons pluto Kuiper belt mission: An overview with historical context, Space Sci. Rev., 140, 3-21, doi:10.1007/s11214007-9295-y, 2008

Tuzzolino, A. J., Two-dimensional position-sensing PVDF dust detectors for measurement of dust particle trajectory, velocity, and mass, Nucl. Instr. Meth. Phys. Res. A, 301, 558-567, doi:10.1016/01689002(91)90023-J, 1991.

Tuzzolino, A. J., PVDF copolymer dust detectors: Particle response and penetration characteristics, Nucl. Instr. Meth. Phys. Res. A, 316, 223237, doi:10.1016/0168-9002(92)90904-I, 1992.

Tuzzolino, A. J., Applications of PVDF dust sensor systems in space, Adv. Space Res., 17, 123-132, doi:10.1016/0273-1177(95)00769-B, 1996.

Vitense, C., A. V. Krivov, and T. Löhne, The Edgeworth-Kuiper debris disk, Astron. Astrophys., 520(A32), doi:10.1051/00046361/201014208, 2010.

Vitense, C., A. V. Krivov, H. Kobayashi, and T. Löhne, An improved model of the Edgeworth-Kuiper debris disk, Astron. Astrophys., 540(A30), doi:10.1051/0004-6361/201118551, 2012.

Yamamoto, S. and T. Mukai, Dust production by impacts of interstellar dust on Edgeworth-Kuiper Belt objects, Astron. Astrophys., 329, 785$791,1998$.

J. R. Szalay, M. Piquette, and M. Horányi (e-mail: horanyi@colorado.edu) 